\title{
Teaching and Practice of Mathematical Modeling
}

\author{
Wenhao Xie \\ Department of Science, Xi'an Shiyou University,Xi'an, China \\ 1609632928@qq.com
}

\begin{abstract}
Keywords: Mathematical Modeling; Teaching structure; Modeling training; Model Test; Linear programming
\end{abstract}

\begin{abstract}
This paper has analyzed and researched the relationship of the mathematical quality-oriented education and mathematical modeling education.It also has deeply analyzed the characteristics and theoretical based on the college students' mathematical modeling teaching and discussed the mathematical modeling teaching content, teaching methods and teaching mode. It has proposed an innovative and integrated teaching model of combing the normal mathematical teaching with the mathematical modeling and mathematical experiments. At last,this paper presents a teaching strategy for the mathematical modeling and discusses how to improve the modeling thinking and modeling ability by the analysis of the examples.
\end{abstract}

\section{Introduction}

Mathematical modeling is the best method of applying the mathematical knowledge into the practical ability. It is also a kind of innovative scientific method. It will simplify the practical problems and abstract them into the mathematical problems or mathematical models, then use the appropriate mathematical methods for solving the solutions and make quantitative analysis and research for the practical problems in order to solving them. It will stimulate the students' curiosity, cultivate the students' innovation consciousness and explore their initiative.[1] At the same time, It has put forward a new topic for our teaching: how to convert the knowledge into the tools of solving the practical problems, how to cultivate the students' ability of acquiring new knowledge and how to use the mathematical modeling education to effectively raise students' mathematical quality [2].

\section{Teaching Structure and Teaching Methods of Mathematical Modeling}

Teaching Structure of Mathematical Modeling. The teaching of mathematical modeling course is a systematic project which involves many branches of mathematics. The teaching structure consists mainly of the following modules.

Basic Knowledge of Mathematics. It includes calculus, linear algebra, ordinary differential equations, probability theory and so on, which is the basic mathematics knowledge that students should master. The basic mathematical knowledge is theoretical for the students[3].

Applied Knowledge of Mathematics. It includes linear programming, graph theory, combinatorial mathematics, operations research, mathematical statistics, mathematical experiments and calculation methods and so on. It is a mathematical method of solving specific problems that students need to grasp based on familiar with the above basic mathematics knowled ge. For example, linear programming is an important branch of operations research. It has rapid development, wide application and mature methods. It is a mathematical theory and method which can assist people to carry on scientific management and study the extermal problems of the linear objective functions under the linear constraint conditions. So the curriculums can combine the mathematical theories with the practical application [4].

Modeling Knowledge. The modeling knowledge includes: Basic methods and common tools for mathematical modeling, mathematical modeling and discussion, modeling training.

"Basic methods and common tools for mathematical modeling" mainly introduces the commonly used mathematical models in the modeling process, some mathematical software and statistical 
software. We should emphasize the training of computer simulation, data fitting, statistical analysis and so on in the course teaching.

"Mathematical modeling and discussion" is the key of the course. Mathematical modeling has its own characteristics in many aspects such as knowledge structure, thinking mode and ability training. Its main purpose is how to build model. We should pay attention to choose the distinctive model which has the features such as particularity practicability, advancement and the integrity.

"Modeling training" is mainly placed in the later stage of modeling teaching. We should divide the students into groups, train the whole process according to the requirements of mathematical modeling, and simulate the mathematical modeling competition. At the same time, we also provide reference for the selection of players in the competition.

The Process and Method of Mathematical Modeling. The process and methods of mathematical modeling are shown as the following [5]

The Actual Situation. Before establishing the mathematical model, it is necessary to understand the situation of the actual problems and master the relevant background knowledge and data information of the problems. Based on analyzing the relationship and specific requirements of the actual problems, the important factors influencing the practical problems should be found and the mathematical knowledge and methods should be firmly grasped by us.

Putting Forward Problems. Establishing the mathematical model is the scientific abstract process that should concretely analyze the practical problems. On the basis of analyzing the actual problems, we abstract and raise questions. It is a process that can convert difficulty to facility. Therefore, we should grasp the main contradiction of the problems, discard the secondary aspect and guess the relationship between the important factors. At last, we simplify this process.

Establishing the Mathematical Model. Based on the hypothesis, the relationship between the numbers of problems is expressed by using the appropriate mathematical methods and establishing the corresponding mathematical model.

Model Solutions and Model Analysis. After modeling, the model should be solved. For example, we can conclude the preliminary mathematical results by means of the solutions of the equations, list, drawing and so on. Then we should analyze, translate and explain the results and point out the actual meaning of the results and the applied range of the model by all kinds of ways such as analyzing the relationship between the variables[6].

Model Test. By applying the results of the model to the actual problems and operating the model, we can compare with the results of the model and the actual situation to verify the reliability and accuracy of the model. For the cases that don't conform to the actual situation, we should adjust it and further put forward the questions.

The Conclusions. For the conclusions that conform to the actual situation, the results are available. After the mathematical model is accepted, it can be applied to the practical application. Then the models should be constantly improved in the practice[7].

\section{The Reform and Practice of Mathematical Modeling Teaching}

Combing in the Ideas of Mathematics Modeling with the Usual Teaching. In the teaching of basic mathematics, we should attach importance to the ideas of modeling.For example, in higher mathematics, we've all learned the chapter of "Ordinary Differential Equations". When we explain this chapter, we can discuss the practical application of mathematical modeling by the example of commodity price.[8]

We use the mathematical models to show the relationship between the price of products and market demand. Firstly, let's set time with $t$ and the price of the products with $P(t) . P(t)$ does not equal the price positioning of the manufacturers. At this time, once the supply and demand relationship of the market changes, the imbalance of supply and demand will cause the price of products to be made again. Under the new pricing, the supply and demand relationship will change again and again. Because of the change in supply and demand relationship, the price of products is constantly changing and dynamic. We found the price of the products $P(t)$ and the ratio of supply 
and demand $\frac{d p}{d t}$ has a positive correlation. We use $f(p, r)$ to represent the market demand and use $g(p)$ to represent the number of products supplied by the market ( $r$ is set value).Such, we have the following formula Eq.1.

$$
\left\{\begin{array}{l}
\frac{d p}{d t}=\alpha[f(p, r)-g(p)] \\
p(0)=p_{0}
\end{array}\right.
$$

Here, $p_{0}$ is the price when $t=0$ and $\alpha>0$.

From here, we can see that the model of market price is a mathematical model of first-order ordinary differential equation, which can be solved by the numerical solution methods of ordinary differential equations[9].

Emphasis on the Reforms of Teaching Methods and Teaching Content. We should attach importance to selecting the cases in the teaching. The excellent cases should be selected according to the teaching sections. We should actively develop the multimedia courseware in teaching and try to break the monotonous teaching mode which is used to impart knowledge as single mode. The students should have the opportunities to participate in teaching and participate in operation. We can apply the test questions of the mathematical modeling competition and the typical cases into our teaching.For example, when we lecture the transportation scheduling and optimization knowledge, we can combine with the title " the vehicles arrangement of open pit mine production ".Then we can apply the vehicles arrangement of open-pit mine production model into the research of transport scheduling problems such as the distribution of supermarket goods and the logistics management and so on. [10]

Cultivating the Cooperative Spirit of the Students and Paying Attention to the Mathematical Experiments. We should pay attention to cultivate the collaboration spirit of the students and teach the students learn to listen to others. We should set up mathematical experiments for a certain part of teaching content, arrange the experiments task beforehand and explain the experiments goals. The students will analyze and probe the problems so as to solve them under the guidance of teachers. By this way, it can arouse their interests in learning.

Attaching Great Importance toTalent Selection. We should be guided by the quality education spirit to select the students and can not only consider the students' examinatorial scores. We should pay attention to the thinking ways, the analyzing and solving problems abilities of the students. We should also pay attention to the diversity and openness of the questions in the selection process so that students can find more information to write the papers. Thus we can better examine the students' comprehensive abilities and quality.

\section{Summary}

In a word, if we can pay attention to deep researching of the teaching content, teaching methods, the teaching mode, and the deep reforms of the mathematical modeling teaching methods, it must effectively improve the students' abilities of analyzing problems and solving problems and arouse the students' interests of learning. Only in this way, the true goals of the quality education can be obtained.

\section{References}

[1] XiuJuan Cao, Xiaoyan Liu, Yanying Wang, Project Results, Teaching and practice of mathematical modeling, Vol 7. (2012), NO.5, P.130-131.

[2] Jun Lu, Higher Science Education, Study on teaching reforms of mathematical main courses based on mathematical modeling, Vol 4. (2014), NO.4, P.20-23.

[3] Xiaoli Li, Journal of Railway Normal University, Vol 9.(2010),NO.2,P.83-84. 
[4] Dongyan Chen, Mathematical Modeling, Beijing,2014.

[5] Lu Zhang, Yuting Li, Zhijun Cheng,Modern commercial industry,Vol.22(2012)No. 3 ,220-221.

[6] Shoukui Si, Liang Sun, Mathematical Modeling Algorithms and Application, Beijing,2015.

[7] Jinwu Zhuo, The Application of Matlab in Mathematical Modeling, Beijing,2014.

[8] Lu Dao, Knowledge Tribune, Vol 6.(2016) No.2 ,P.97-98.

[9] Jianwei Du, Ruopeng Wang, Basic Cases of Mathematical Modeling, Beijing, 2014.

[10] Chun Cai, Hongmin Song,Qinghua Ma. Journal of management education, Vol 8. (2008) No.1, P.87-88. 